\title{
Some exact analytic results for the linear and non-linear response of atoms in a trap with a model interaction
}

\author{
Simon C. Benjamin ${ }^{1}$, Luis Quiroga ${ }^{2}$ and Neil F. Johnson ${ }^{1}$ \\ ${ }^{1}$ Department of Physics, Oxford University, Oxford, OX1 3PU, England \\ ${ }^{2}$ Departamento de Fisica, Universidad de Los Andes, Bogota, A.A. 4976, Colombia
}

\begin{abstract}
We present an exact expression for the evolution of the wavefunction of $N$ interacting atoms in an arbitrarily time-dependent, $d$-dimensional parabolic trap potential $\omega(t)$. The interaction potential between atoms is taken to be of the form $\xi / r^{2}$ with $\xi>0$. For a constant trap potential $\omega(t)=\omega_{0}$, we find an exact, infinite set of relative mode excitations. These excitations are relevant to the linear response of the system; they are universal in that their frequencies are independent of the initial state of the system (e.g. Bose-Einstein condensate), the strength $\xi$ of the atom-atom interaction, the dimensionality $d$ of the trap and the number of atoms $N$. The time evolution of the system for general $\omega(t)$ derives entirely from the solution to the corresponding classical 1D single-particle problem. An analytic expression for the frequency response of the $N$-atom cluster is given in terms of $\omega(t)$. We consider the important example of a sinusoidally-varying trap perturbation. Our treatment, being exact, spans the 'linear' and 'non-linear' regimes. Certain features of the response spectrum are found to be insensitive to interaction strength and atom number.
\end{abstract}

PACS numbers: $32.80 . P j, 42.50 . V k, 67.65 .+z$ 


\section{INTRODUCTION}

Atomic traps have been the subject of much recent research activity, both theoretical [1] and experimental [2] [3]. The finite atom number $N$ and reduced effective dimensionality $d \leq 3$ in atomic traps opens up a fascinating research area of confined $N$-atom states. A singularly important milestone in this field has been the recent experimental observation of a Bose-Einstein condensate in an effectively two-dimensional $(d=2)$ trap [2]. It is to be expected that the application of time-dependent perturbations to such traps will provide useful information as to the properties of the $N$-atom system.

According to the preparation of the atomic trap, the confinement length scales in the three spatial directions $\left(L_{x}, L_{y}\right.$ and $\left.L_{z}\right)$ can, in principle, be quite different yielding highly anisotropic traps. Within a simple particle-in-a-box picture the single-particle energy level spacing $\Delta E \sim L^{-2} ;$ it follows that for $L_{x}>>L_{y}, L_{z}$ the atoms will be stuck in the lowest $y, z$ subbands hence freezing out the $y, z$ degrees of freedom. The anisotropic three-dimensional trap is now effectively quasi-one-dimensional in that the atoms only have significant freedom along the $x$-direction. Similarly if $L_{x} \sim L_{y}>>L_{z}$ the trap is effectively quasi-twodimensional; if $L_{x} \sim L_{y} \sim L_{z}$ the trap becomes quasi-three-dimensional.

A variety of theoretical predictions have been made concerning the properties of atomic traps. For example, Burnett and co-workers have obtained very interesting results concerning the linear and non-linear responses of trapped neutral atoms in a Bose-Einstein condensate by solving a time-dependent Gross-Pitaevskii equation for the condensate order-parameter or 'wavefunction' [四]. Important open questions concern the precise dependence of the $\mathrm{N}$ atom quantum state on the number of atoms $N$ [5], and on the strength of the interaction between atoms [6].

Given the general intractability of the $N$-body problem, most theoretical approaches have been numerical and tend to be based on macroscopic, phenomenological models or meanfield approximations. Our results in this paper complement such studies. In particular we provide analytic results for the response of an arbitrary number $N$ of trapped, neutral atoms by studying a microscopic model Hamiltonian. We provide closed-form expressions 
for the trap properties as a function of the trap parameters, thereby yielding insight into the competition between the confinement and atom-atom interactions. The analytic tractability of our model is made possible through a combination of the parabolic form for the confining potential in the magnetic traps of interest [1], and an inverse-square interaction between atoms, i.e. $1 / r^{n}$ with $n=2$. The actual short-range repulsive interaction between neutral atoms is likely to be better fitted by a larger $n$; however, the general features of our results should be qualitatively similar for other short-range interactions involving $n>2$.

The paper is organized as follows. In Sec. II we discuss the excitation spectrum obtained from the time-independent $N$-atom Schrodinger equation for an isolated trap. These excitations are relevant for describing the response of the trap to a weak, sinusoidal perturbation (i.e. linear response). In Sec. III we consider a class of time-dependent perturbations having arbitrary strength and form, and derive the exact, time-dependent $N$-atom wavefunction. As an example, the exact, non-linear time-dependent response to a sinusoidal perturbation is developed in Sec. IV; this response is shown to be largely determined by solutions of the well-known Mathieu equation. Non-linear features in the system response are identified and explained. Throughout the paper we will focus on quasi-two-dimensional traps, since these systems are attracting much experimental interest. The generalization of these results to quasi-one-dimensional and isotropic, three-dimensional traps is straightforward and the corresponding results are discussed briefly. We will also focus on the case of repulsive interactions between atoms. The extent to which our results are also applicable to attractive atom-atom interactions is discussed in Sec. V, together with the conclusions. We will comment throughout on the extent to which our analytic results can reproduce similar physics to recent numerical work.

\section{ELEMENTARY EXCITATIONS OF AN ISOLATED TRAP}

In this section we focus on the elementary excitations of an isolated trap. Such excitations govern the linear response of the system in the presence of a weak, sinusoidal perturbation. We will specifically obtain an exact, infinite set of relative mode excitations for the $N$-atom 
system.

The governing microscopic equation for the energy spectrum of the atom trap is the $N$ atom non-relativistic Schrodinger equation with a $d$-dimensional confining potential $V_{C}(\mathbf{r})$; this is given by $H \Psi=E \Psi$ with

$$
H=\sum_{i}\left[\frac{1}{2 m} \mathbf{p}_{i}^{2}+V_{C}\left(\mathbf{r}_{i}\right)\right]+\sum_{i<j} V_{I}\left(\mathbf{r}_{i}-\mathbf{r}_{j}\right),
$$

where the momentum and position associated with the $i$ 'th atom are given by $\mathbf{p}_{i}$ and $\mathbf{r}_{i}$. The magnetic traps of interest have approximately parabolic confining potentials $V_{C}$. We will assume that the trap potential for a $d$-dimensional trap is a $d$-dimensional isotropic harmonic oscillator, ie. $V_{C}=\frac{1}{2} m \omega_{0}^{2} r_{i}^{2}$. We also assume the atom-atom interaction is translationallyinvariant. The exact functional form of the two-body interaction $V_{I}\left(\mathbf{r}_{i}-\mathbf{r}_{j}\right)$ between neutral atoms is unknown; here we focus on a net repulsive interaction between atoms of the form $\xi\left(\left|\mathbf{r}_{i}-\mathbf{r}_{j}\right|\right)^{-n}$ where $n=2$ and $\xi>0$. Note that for $n \geq 2$, the interaction is sufficiently 'short-range' that we would expect similar behavior to emerge for a range of $n$. At the end of the paper we briefly discuss how our results carry over to an attractive interaction between atoms (i.e. $\xi<0$ ).

We employ standard Jacobi coordinates $\mathbf{X}_{i}(i=0,1, \ldots, N-1)$ where $\mathbf{X}_{0}=\frac{1}{N} \sum_{j} \mathbf{r}_{j}$ (center-of-mass), $\mathbf{X}_{1}=\sqrt{\frac{1}{2}}\left(\mathbf{r}_{2}-\mathbf{r}_{1}\right), \mathbf{X}_{2}=\sqrt{\frac{2}{3}}\left(\frac{\left(\mathbf{r}_{1}+\mathbf{r}_{2}\right)}{2}-\mathbf{r}_{3}\right)$ etc. together with their conjugate momenta $\mathbf{P}_{i}$. The center-of-mass motion decouples, $H=H_{\mathrm{CM}}\left(\mathbf{X}_{0}\right)+H_{\text {rel }}\left(\left\{\mathbf{X}_{i>0}\right\}\right)$, hence $E=E_{\mathrm{CM}}+E_{\mathrm{rel}}$ and $\Psi=\psi_{\mathrm{CM}} \psi_{\text {rel }}$. The exact eigenstates $\psi_{\mathrm{CM}}$ of $H_{\mathrm{CM}}$ and eigenenergies $E_{\mathrm{CM}}$ are identical to those of a single particle in a parabolic potential and are well-known. We will concentrate on the relative motion since it is here that effects of atom-atom correlations manifest themselves. The non-trivial problem is to solve the relative motion equation $H_{\text {rel }} \psi_{\text {rel }}=E_{\text {rel }} \psi_{\text {rel }}$. We transform the relative coordinates $\left\{\mathbf{X}_{i>0}\right\}$ to standard hyperspherical coordinates: $\mathbf{X}_{i}=r\left(\prod_{j=i}^{N-2} \cos \alpha_{j+1}\right) \sin \alpha_{i} e^{i \theta_{i}}$ with $r \geq 0$ and $0 \leq \alpha_{i} \leq \frac{\pi}{2}\left(\alpha_{1}=\frac{\pi}{2}\right)$. Physically, the hyperradius $r$ is related to the root-mean-square atom-atom separation:

$$
r^{2}=\sum_{j=1}^{N-1} X_{j}^{2}=\frac{1}{N} \sum_{\left(i>i^{\prime}\right)}^{N}\left(\mathbf{r}_{i}-\mathbf{r}_{i^{\prime}}\right)^{2}
$$


As mentioned above, we will focus on $d=2$. The exact eigenstates of $H_{\text {rel }}$ have the form $\psi_{\text {rel }}=R(r) F(\tilde{\Omega})$ where $\tilde{\Omega}$ denotes the $(2 N-3)$ remaining $\{\theta, \alpha\}$ variables; $R(r)$ and $F(\tilde{\Omega})$ are solutions of the hyperradial and hyperangular equations respectively. The hyperradial equation is

$$
\left(\frac{d^{2}}{d r^{2}}+\frac{2 N-3}{r} \frac{d}{d r}-\frac{\gamma(\gamma+2 N-4)}{r^{2}}-\frac{r^{2}}{l_{0}^{4}}+\frac{2 m E_{\mathrm{rel}}}{\hbar^{2}}\right) R(r)=0
$$

where $l_{0}^{2}=\hbar\left(m \omega_{0}\right)^{-1}$; the parameter $\gamma$ is related to the eigenvalue of the $\omega_{0}$-independent hyperangular equation (see below). Equation (3) can be solved exactly yielding

$$
E_{\mathrm{rel}}=\hbar \omega_{0}(2 n+\gamma+N-1)
$$

where $n$ is any positive integer or zero and

$$
R_{n}(r)=\left(\frac{r}{l_{0}}\right)^{\gamma} L_{n}^{\gamma+N-2}\left(\frac{r^{2}}{l_{0}^{2}}\right) e^{-\frac{r^{2}}{2 l_{0}^{2}}} .
$$

where $L$ is the Laguerre Polynomial. Equation (4) provides an exact (and infinite) set of relative mode excitations at frequencies $2 \omega_{0} \Delta n$, irrespective of the initial eigenstate of the $N$-atom system (e.g. Bose-Einstein condensate). These are 'breathing' modes; we note that they are very similar in frequency to the set of modes found to occur near multiples of $2 \omega_{0}$ in recent numerical calculations based on the Gross-Pitaevskii equation [4.

The $\omega_{0}$-independent hyperangular equation determining $\gamma$ is given by

$$
\left[\Theta_{N}^{2}+\frac{2 m \xi}{\hbar^{2}} V(\tilde{\Omega})\right] F(\tilde{\Omega})=[\gamma(\gamma+2 N-4)] F(\tilde{\Omega})
$$

where

$$
\Theta_{N}^{2} \equiv-\frac{\partial^{2}}{\partial \alpha_{N}^{2}}+\frac{\left[2 N-6-(2 N-4) \cos 2 \alpha_{N}\right]}{\sin 2 \alpha_{N}} \frac{\partial}{\partial \alpha_{N}}+\sec ^{2} \alpha_{N} \Theta_{N-1}^{2}-\operatorname{cosec}^{2} \alpha_{N} \frac{\partial^{2}}{\partial \theta_{N}^{2}}
$$

The quantity $V(\tilde{\Omega})$ represents the hyperangular part of the atom-atom interaction and is given by

$$
V(\tilde{\Omega})=r^{2} \sum_{i<j} \frac{1}{\left|\mathbf{r}_{i}-\mathbf{r}_{j}\right|^{2}}
$$


Since the atom-atom interaction only depends on absolute relative coordinates, $V(\tilde{\Omega})$ commutes with the relative angular momentum. The relative angular momentum $J$ therefore remains a good quantum number, hence we can introduce a further Jacobi transformation of the relative motion angles $\left\{\theta_{i}\right\}$ : in particular $\theta^{\prime}=\frac{1}{N-1} \sum_{i=1}^{N-1} \theta_{i}, \theta=\frac{1}{\sqrt{2}}\left(\theta_{1}-\theta_{2}\right)$ etc. The hyperangular equation now depends on just $(2 N-4)$ remaining hyperangles, i.e. the hyperangles $\tilde{\Omega}$ excluding $\theta^{\prime}$. We denote these $(2 N-4)$ hyperangles as $\Omega$. The exact eigenstates of $H_{\text {rel }}$ now have the form $\psi_{\text {rel }}=e^{i J \theta^{\prime}} R(r) G(\Omega)$ (i.e. $F(\tilde{\Omega})=e^{i J \theta^{\prime}} G(\Omega)$ ).

Unfortunately the hyperangular equation (Eq. (6)) does not admit complete exact solutions for $\gamma$ and $F(\tilde{\Omega})$, or equivalently $G(\Omega)$. However some insight into the properties of these eigenstates can be gained as follows. Without loss of generality, we can choose to rewrite the eigenvalue of the hyperangular equation (Eq. (6)) in terms of a new quantity $\epsilon$ as

$$
\epsilon=\frac{\hbar^{2}}{8}\left[\gamma(\gamma+2 N-4)-\left(\frac{V_{\text {class }}}{\hbar \omega_{0}}\right)^{2}-J^{2}\right]
$$

where $V_{\text {class }}$ is the potential energy of the minimum-energy configuration for classical atoms in the trap (i.e. the Wigner solid). This quantity $\epsilon$ is useful in that it isolates purely quantum-mechanical contributions to $\gamma$, i.e. it does not contain either the classical potential energy of the atoms or their rotational kinetic energy. It is straightforward to show that $V_{\text {class }} \propto \xi^{\frac{1}{2}} \omega_{0}$ and hence $\epsilon$ (like $\gamma$ ) is independent of $\omega_{0}$. We can now recast the exact relative energy expression as

$$
E_{\text {rel }}=\hbar \omega_{0}\left[2 n+\left([N-2]^{2}+J^{2}+\left(\frac{V_{\text {class }}}{\hbar \omega_{0}}\right)^{2}+\frac{8 \epsilon}{\hbar^{2}}\right)^{\frac{1}{2}}+1\right] .
$$

$E_{\text {rel }}$ only depends on particle statistics through $\epsilon$. As $\hbar \rightarrow 0, E_{\text {rel }} \rightarrow V_{\text {class }}$ and $\epsilon \rightarrow$ 0 . Physically, $\epsilon$ includes the zero-point energy in $\Omega$-space associated with the quantummechanical spread of $G(\Omega)$ about the hyperangles $\Omega$ corresponding to the classical, minimum energy configuration of the $N$-atom system (Wigner solid). In the limit of fairly weak interatomic interactions (i.e. small $\xi$ ), the spread in $G(\Omega)$ and hence the magnitude of the zero-point energy $\epsilon$ will be large. For any given $\xi$, the spread in $G(\Omega)$ and hence magnitude of 
$\epsilon$ will also depend on total wavefunction symmetry requirements; $\epsilon$ will in general be smaller for a bosonic ground state than for a fermionic ground state due to the lower number of nodes in $G(\Omega)$ for bosons.

The above analysis which yielded the exact excitation frequencies $2 \omega_{0} \Delta n$ for quasi-twodimensional $(d=2)$ traps carries over to quasi-one-dimensional traps $(d=1)$ and isotropic three-dimensional traps $(d=3)$. The same breathing-mode frequencies $2 \omega_{0} \Delta n$ are obtained in each case. For $d=1$ the quantity $\gamma$ in Eq. (4), and hence the entire energy spectrum $E_{\text {rel }}$, can actually be obtained analytically. This model was first solved exactly by Calogero [7]. The exact $N$-atom energy levels for a quasi-one-dimensional trap are given by $E=E_{\mathrm{CM}}+E_{\text {rel }}$ with

$$
E_{\mathrm{rel}}=\hbar \omega_{0}\left(\frac{1}{2}(N-1)+\frac{1}{2} N(N-1)\left(\tau+\frac{1}{2}\right)+k\right)
$$

where $\tau=\frac{1}{2}\left(1+\frac{4 m \xi}{\hbar^{2}}\right)^{\frac{1}{2}}$ and $k$ is a positive integer (N.B. $\left.k \neq 1\right)$. The hyperradial breathing modes $2 \hbar \omega_{0} \Delta n$ are included in this relative mode spectrum via the integer $k$ values. We note that Eq. (11), while exact for non-zero $\xi$, does not yield the full energy level spectrum for $N$ non-interacting atoms in the limit $\xi \rightarrow 0$. This feature, which is discussed explicitly in Ref. [7], results from the singular nature of the inverse-square interaction when applied in one dimension - it does not arise in two or three dimensions.

\section{DYNAMICS OF A PERTURBED TRAP}

In this section we extend the above results to include a time-dependent trap potential

$$
\omega^{2}(t)= \begin{cases}\omega_{0}^{2} & \text { for } t \leq 0 \\ f(t) & \text { for } t>0\end{cases}
$$

The treatment will be exact, analytic and applicable to any function $f(t)$ with arbitrarily large magnitude, i.e. we implicitly include all non-linear effects to all orders. Specifically, we will provide the time-dependent versions of the hyperradial breathing-mode wavefunctions given in Eq. (5). These will be found to depend only on the solution of a classical onedimensional oscillator with trap potential $\omega^{2}(t)$. Again we will specifically consider a quasi- 
two-dimensional $(d=2)$ trap, but the method is equally applicable to both $d=1$ and $d=3$.

The time-dependent Schrodinger equation is given by $H \Psi=i \hbar(\partial \Psi / \partial t)$. In the presence of the time-dependent trap potential discussed above, the separation of the center-of-mass and relative motion is still exact, $\Psi=\psi_{\mathrm{CM}}(t) \psi_{\text {rel }}(t)$. It was found in Sec. II that the hyperangular equation is independent of $\omega_{0}$, hence those parts of the relative wavefunction $\psi_{\text {rel }}$ that derive from this equation remain time-independent. The time-dependence of the total wavefunction $\Psi$ is therefore only contained in the center-of-mass and hyperradial parts. Here we consider explicitly $\psi_{\text {rel }}(t)$ and hence the hyperradial part $R(r, t)$. The solution for the trivial center-of-mass part $\psi_{\mathrm{CM}}(t)$ is exactly analogous. Following $[8$ we construct the generating function

$$
g(z, r, t) \equiv \sum_{n=0}^{\infty} R_{n}(r, t) z^{n}
$$

where $R_{n}(r, t)$ are the solutions of the time-dependent hyperradial equation. Because the trap potential is constant for $t \leq 0$, we can employ Eq. (5) to obtain

$$
g(z, r, t \leq 0)=\sum_{n=0}^{\infty}\left(\frac{r}{l_{0}}\right)^{\gamma} L_{n}^{\gamma+N-2}\left(\frac{r^{2}}{l_{0}^{2}}\right) e^{-\frac{r^{2}}{2 l_{0}^{2}}} z^{n}
$$

This can be written in closed form using the identity

$$
\sum_{n=0}^{\infty} z^{n} L_{n}^{a}(y) \equiv(1-z)^{-(a+1)} e^{\left(\frac{z y}{z-1}\right)}
$$

(see Ref. 91) so that

$$
g(z, r, t \leq 0)=\left(\frac{r}{l_{0}}\right)^{\gamma} e^{\frac{z+1}{2(z-1)}\left(\frac{r}{l_{0}}\right)^{2}}(1-z)^{-(a+1)}
$$

where $a=\gamma+N-2$. We now make the ansatz

$$
g(z, r, t>0)=\alpha(z, t)\left(\frac{r}{l_{0}}\right)^{\gamma} e^{\alpha^{\prime}(z, t) r^{2}}
$$

which can be shown to satisfy the time-dependent hyperradial equation

$$
\left(\frac{d^{2}}{d r^{2}}+\frac{2 N-3}{r} \frac{d}{d r}-\frac{\gamma(\gamma+2 N-4)}{r^{2}}-\left(\frac{m}{\hbar}\right)^{2} \omega^{2}(t) r^{2}\right) g(z, r, t)=-\frac{2 i m}{\hbar} \frac{\partial}{\partial t} g(z, r, t)
$$


and the $t=0$ boundary condition in Eq. (16), provided

$$
\alpha(z, t)=[\eta(t)]^{-(a+1)} \exp [2 i \theta(t)(a+1)](1-z \exp [2 i \theta(t)])^{-(a+1)}
$$

and

$$
\alpha^{\prime}(z, t)=\frac{i m}{2 \hbar}\left(\frac{\dot{\eta(t)}}{\eta(t)}-2 i \dot{\theta}(t)(1-z \exp [2 i \theta(t)])^{-1}\right)
$$

where $\eta(t)=|\eta(t)| e^{i \theta(t)}$ solves the classical one-dimensional oscillator

$$
\ddot{\eta}(t)+f(t) \eta(t)=0
$$

with boundary conditions $\eta(0)=1$ and $\dot{\eta(0)}=-i \omega_{0}$. We may then expand $g(z, r, t)$ using the identity (15), and compare coefficients of $z^{n}$ with the defining Eq. (13) to obtain the desired time-dependent wavefunctions (unnormalised):

$$
R_{n}(r, t>0)=|\eta(t)|^{1-N} y^{\gamma} \exp \left[i\left(\theta(t)(2 n+a+1)+\frac{y^{2}}{4 \omega_{0}} \frac{d}{d t}|\eta(t)|^{2}\right)\right] e^{-\frac{1}{2} y^{2}} L_{n}^{a}\left(y^{2}\right)
$$

where $y \equiv \frac{r}{|\eta| l_{0}}$. In the static case this expression reduces to equation (5). If for a particular $f(t)$ we are able to solve Eq. (21), then Eq. (22) provides a complete description for the evolution of the initially stationary hyperradial state $R_{n}$ as the trap becomes timedependent for $t>0$. As noted previously, since the remaining hyperangular part of $\psi_{\text {rel }}$ is time-independent, Eq. (22) together with the (exactly analogous) expression for the centerof-mass entirely determines the time-evolution of the total wavefunction $\Psi=\psi_{\mathrm{CM}}(t) \psi_{\text {rel }}(t)$.

We now comment on the significance of our results, particularly with respect to the Bosecondensed atomic gas. Our results for the breathing modes in Sec. II and for the dynamical response in Sec. III are valid for all strengths of the atom-atom interaction $(\xi)$, all trap sizes $\left(\omega_{0}\right)$ and all numbers of atoms $(N)$. They are independent of the precise form of the initial state of the system and are therefore true in both the Bose-condensed and non-condensed regimes. Since we do not know $\gamma($ or $F(\tilde{\Omega}))$ explicitly, our model does not allow us to predict a temperature at which the gas will be substantially Bose-condensed. This is consistent with the expectation that the condensation temperature has a complicated dependence on $\omega_{0}, \xi$ and $N$. 
In deriving the time-dependent wavefunction of Sec. III, we took the $\mathrm{N}$-atom system to be in a single eigenstate of the time-independent Schrodinger equation up until the moment the perturbation is turned on (i.e. for $t<0$ ). The linearity of the Schrodinger equation implies that any initial wavefunction consisting of a sum of eigenstates will evolve as the sum of its parts, and may therefore be written as a sum of terms such as Eq. (22). In Sec. IV below, the calculated response spectrum is also derived under the assumption of a single eigenstate for $t<0$, however the initial state can be generalized to a sum of eigenstates without altering the response spectrum. The results we present in this paper are therefore remarkably general in their applicability.

\section{EXAMPLE: SINUSOIDAL PERTURBATION}

As an illustration of the applicability of our formalism, we now take a particular $f(t)$ and employ Eq. (22) to calculate the frequency response spectrum of the system. We find that the spectrum exhibits some features which are essentially independent of $\gamma$. Since the $\xi$-dependence only enters the response through $\gamma$, it follows that any observables found to be substantially independent of $\gamma$ are generic to all atom traps independent of the strength of the atom-atom interaction $\xi$.

We consider the specific case of a sinusoidal perturbation turned on at $t=0$ :

$$
\omega^{2}(t)= \begin{cases}\omega_{0}^{2} & \text { for } t \leq 0 \\ f(t)=\omega_{0}^{2}-\omega_{1}^{2}(1-\cos (2 \Omega t)) & \text { for } t>0\end{cases}
$$

A sketch of this function appears at the top of Fig. 1. It would be quite possible to produce this trap perturbation experimentally. The solution to the classical equation (Eq. (21)) is a general Mathieu function, which may be written in the form

$$
\eta(t)=A e^{\mu \Omega t} \sum_{n=-\infty}^{\infty} c_{2 n} e^{2 i n \Omega t}+B e^{-\mu \Omega t} \sum_{n=-\infty}^{\infty} c_{2 n} e^{-2 i n \Omega t}
$$

where $\mu$ and $\left\{c_{2 n}\right\}$ are determined by a set of simultaneous equations (see Ref. [9]).

In the main part of Fig. 1 we show the behavior of $\mu$ as a function of the parameters $\omega_{0}$, $\omega_{1}$ and $\Omega$. This behavior is non-trivial; in certain regions (shown white) of the parameter 
space $\mu$ is purely imaginary while in others (shown dark) it has a real part. We define $\beta \equiv \operatorname{Im}\{\mu\}$. A non-zero real part of $\mu$ indicates that the classical particle is resonating with the oscillating trap; the particle's oscillations then become infinitely large as $t \rightarrow \infty$. The corresponding effect on the quantum mechanical system, which depends on time only through $\eta(t)$, will be an increase in energy and a decrease in localization of $R(r, t)$ and hence $\psi_{\text {rel }}$. This spreading in $\psi_{\text {rel }}$ implies an increase in the average atom-atom separation, and will lead to atoms escaping from any realistic trap having a finite depth. For small $\omega_{1}, \mu$ has a real component only when the perturbing frequency $\Omega$ is equal to an integer fraction $\frac{1}{n}$ of the trap potential $\omega_{0}$. We emphasize that our treatment is exact for any amplitude $\omega_{1}^{2}$ of the sinusoidal perturbation. Indeed the theory remains sound even when $\omega_{1}^{2}>\frac{1}{2} \omega_{0}^{2}$, in which case the trap becomes repulsive for part of each oscillation.

In the remainder of this section we concern ourselves with solutions which are 'stable', i.e. points in the parameter space corresponding to a purely imaginary $\mu$. As in the numerical studies of Ref. [4], we will calculate the frequency response spectrum of the atomic gas by Fourier analyzing the temporal variation of the gas density, the 'single-particle-density', at a fixed point in space. We define the single-particle-density at a point $\mathbf{S}$ as

$$
d(\mathbf{S}, t)=\sum_{i=1}^{N} \int\left|\Psi\left(\mathbf{r}_{1}, \mathbf{r}_{2}, \ldots, \mathbf{r}_{i-1}, \mathbf{S}, \mathbf{r}_{i+1}, \ldots \mathbf{r}_{n} ; t\right)\right|^{2} \prod_{j \neq i} d \mathbf{r}_{j}
$$

where $\Psi$ is the total wavefunction at time $t$ which, in our case, exactly separates into centerof-mass, hyperradial and angular parts. If we set $\mathbf{S}=\mathbf{0}$ (i.e. the trap center), we find

$$
d(\mathbf{S}=\mathbf{0}, t)=N \int\left|\psi_{C M}^{\mathbf{S}=\mathbf{0}}(t)\right|^{2}\left|R_{n}(r, t)\right|^{2}|F(\tilde{\Omega})|^{2} r^{2 N-3} d r d \tilde{\Omega}
$$

The superscript on $\psi_{C M}(t)$ indicates that under the $\mathbf{S}=\mathbf{0}$ constraint, the center-of-mass coordinate is no longer independent of the other coordinates. Examining the form of $\psi_{C M}$ and $R$ we find that the integral in Eq. (26) may be rewritten in time-independent form using the scaled variable $y=\frac{r}{|\eta(t)| l_{0}}$, as employed in Eq. (22). This transformation to a time-independent integral results in an external factor $|\eta(t)|^{-2}$, thus the density is found to have a simple time-dependence: 


$$
d(\mathbf{S}=\mathbf{0}, t) \propto|\eta(t)|^{-2} .
$$

Interestingly, this expression (Eq. (27)) is completely general for any time-varying trap $\omega^{2}(t)$; here we have chosen to consider a sinusoidal form for which the corresponding $\eta(t)$ may be found analytically. The expression for $d(\mathbf{S}=\mathbf{0}, t)$ is independent of both $N$ and the unknown quantity $\gamma$, thus the peak positions in the Fourier transform must be independent of variations either in the atom-atom interaction strength $\xi$ or in the number of atoms $N$ confined in the trap. Figure 2 displays the Fourier transform of Eq. (27) calculated with a particular choice of the parameters $\omega_{0}$ and $\omega_{1}$. We see a number of sharply defined peaks; the frequencies at which these peaks occur are all of the form $n \Omega+m \Omega \beta$ where $n$ and $m$ are integers. The value of $\beta$ may be found from Fig. 1; for the present choice of parameters $\beta=1.409$.

Figure 1 also shows that when the perturbation amplitude $\omega_{1}$ is small then $\beta \approx \frac{\bar{\omega}}{\Omega}$, where $\bar{\omega}^{2}=\omega_{0}^{2}-\omega_{1}^{2}$ is the mean trap confinement. Thus for a weak-to-moderate perturbation, the peaks in the response lie at sums and differences of $\bar{\omega}$ and the driving frequency $\Omega$; i.e. at $n \Omega+m \bar{\omega}$. Figure 1 shows us exactly how $\beta$ (and hence the spectrum) changes as we move to the strongly non-linear regime $\left(\omega_{1} \approx \bar{\omega}\right)$. The parameters chosen for Fig. 2 correspond to a 'moderate' perturbation $\left(w_{1}^{2}=0.138 \omega_{0}^{2}\right)$; the peaks marked $n \beta$ in Fig. 2 deviate from $n \omega_{0}$ by about $8 \%$, and from $n \bar{\omega}$ by about $0.3 \%$. Although a direct comparison with the nonlinear response of Ref. [4 is not practical because of the different form of the perturbation (i.e. white noise) it is interesting to note that the same types of non-linear phenomena are observed; in particular, harmonic generation and frequency mixing. As noted in Ref. 四, such non-linear effects open up the possibility of non-linear atom optics based on coherent matter waves.

\section{CONCLUSION}

We have presented an analytically-solvable model of the quantum-mechanical timeevolution of $N$ interacting atoms in an arbitrarily time-dependent, $d$-dimensional parabolic 
trap potential $\omega(t)$. The solution allows us to determine the resonance frequencies of the system in response to both weak (linear response) and strong (non-linear response) perturbations. An exact, infinite set of relative mode excitations were found for a constant trap potential $\omega(t)=\omega_{0}$; these excitations are universal in that their frequencies are independent of the initial state of the system (e.g. Bose-Einstein condensate), the strength $\xi$ of the atomatom interaction, the dimensionality $d$ of the trap and the number of atoms $N$. The specific example of a sinusoidally-varying trap perturbation was employed to demonstrate the formalism. Certain features in the system response were found to be insensitive to interaction strength and atom number.

Finally we will discuss the extent to which our model can describe an attractive interaction between atoms (i.e. $\xi<0$ ). The general formalism using hyperspherical coordinates is still valid; the difference is that $\epsilon$ (see Eq. (9)) can now be negative, hence there is a possibility of collapse of the $N$-atom system if the attraction is too strong (i.e. the atoms collapse to form an infinitely dense gas). Such a complete collapse is an artefact of the inverse-square attractive interaction. It could be prevented by inserting an additional hard-core repulsion into the model, but the solvability of the model would be lost. Consider first a pair of atoms $(N=2)$ with an attractive interaction $(\xi<0)$. In a quasi-one-dimensional trap $(d=1)$, it can easily be shown that two-atom collapse occurs if the strength of the attractive interaction $|\xi|>\frac{\hbar^{2}}{4 m}$; only weak attractive interactions where $|\xi|<\frac{\hbar^{2}}{4 m}$ are therefore described by the present theory. The reason that a pair of atoms with a weak attraction can support a non-collapsed ground state in the trap is that the negative potential energy is offset by a large, positive kinetic energy as a result of the uncertainty principle. In an isotropic three-dimensional trap $(d=3)$, two-atom collapse will occur if the strength of the attractive interaction $|\xi|>\frac{\hbar^{2}}{4 m}(1+4 l(l+1))$ where $l$ is the relative angular momentum of the two atoms. Hence for $l=0$, the same condition holds as for $d=1$. For quasi-two-dimensional traps, it turns out that two-atom collapse will occur for $|\xi|>\frac{\hbar^{2} l_{z}^{2}}{m}$ where $l_{z}$ is the component of relative angular momentum perpendicular to the two-dimensional plane. Therefore, for $l_{z}=0$ an arbitrarily small attractive potential will cause two-atom collapse. For $N$ atoms 
in a quasi-one-dimensional trap, the same condition holds as for two atoms. For quasi-two and three-dimensional traps, the exact condition is not known since the problem is not completely solvable ( $\gamma$ and hence $\epsilon$ are not known exactly for $N>2$ ). It remains to be seen, therefore, whether the increase in kinetic energy during collapse is sufficient to offset the decrease in potential energy. If such a condition were to be satisfied, the $N$-atom system with attractive interactions would form a ground state that was qualitatively different from the $N$-atom system with repulsive interactions. As a matter of interest, we note that a suggestion of possible alternative states for $N$ atoms with attractive interactions $\left({ }^{7} \mathrm{Li}\right)$ has recently appeared [10].

We would like to thank Keith Burnett, Peter Ruprecht and Nikos Nicopoulos for very useful discussions. S.C.B. is supported by an EPSRC studentship. Partial funding was also provided by COLCIENCIAS under Project no. 1204-05-264-94. 


\section{REFERENCES}

[1] For a recent review, see K. Burnett, Contemp. Phys. 37, 1 (1996).

[2] M. Anderson, J.R. Ensher, M.R. Matthews, C.E. Wieman and E.A. Cornell, Science 269, $182(1995)$.

[3] R.J.C. Spreeuw, C. Gerz, Lori S. Goldner, W. D. Phillips, S. L. Rolston, C. I. Westbrook, M. W. Reynolds and Isaac F. Silvera, Phys. Rev. Lett. 72, 3162 (1994); C.J. Foot, Contemp. Phys. 32, 369 (1991).

[4] P.A. Ruprecht, M.J. Holland, K. Burnett and M. Edwards, Phys. Rev. A 514704 (1995); M. Edwards, P.A. Ruprecht, K. Burnett and C.W. Clark (to be published); P.A. Ruprecht, D.Phil dissertation, Oxford University (1995).

[5] S. Grossmann and M. Holthaus, Phys. Lett. A 208, 188 (1995).

[6] Yu. Kagan, G.V. Shlyapnikov and J.T.M. Walraven, Phys. Rev. Lett. 76, 2670 (1996); see also Physics Today, March 1996, p.18.

[7] F. Calogero, J. Math. Phys. 12, 419 (1971).

[8] P. Camiz, A. Gerardi, C. Marchiori, E. Presutti and E. Scacciatelli, J. Math. Phys. 12 2040, (1971).

[9] I.S. Gradshteyn and I.M. Ryzhik, Tables of Integrals, Series and Products (Academic Press, London, 1994).

[10] See Physics Today, March 1996, p.18 and references therein. 


\section{Figure Captions}

Figure 1. Top: The trap potential $\omega^{2}(t)$ (cf. Eq.(23)). Bottom: Contour plot showing the behavior of the constant $\mu$ in the Mathieu function $\eta(t)$ (Eq. (21)) as a function of the trap potential parameters. This quantity $\mu$ is complex, $\mu=\operatorname{Re}\{\mu\}+i \beta$. White regions: $\operatorname{Re}\{\mu\}=0$ and the contours are lines of constant $\beta$; for $\omega_{1} \rightarrow 0, \beta \rightarrow \frac{\bar{\omega}}{\Omega}$. Dark regions: $\operatorname{Re}\{\mu\}>0$ and the contours are lines of constant $\operatorname{Re}\{\mu\}$ in increments of 0.2 .

Figure 2. Frequency response spectrum showing Fourier amplitude as a function of frequency response for a trap with parameters $\omega_{1}^{2}=0.138 \omega_{0}^{2}$ and $\Omega^{2}=0.431 \omega_{0}^{2}$. For these parameters $\beta=1.41$; the $\beta$ for any particular trap parameters $\left(\omega_{0}, \omega_{1}, \Omega\right)$ may be found using Fig. 1 . Note that the two central lines (shown as cut-off) exceed the height of the graph by a factor of 3 . 

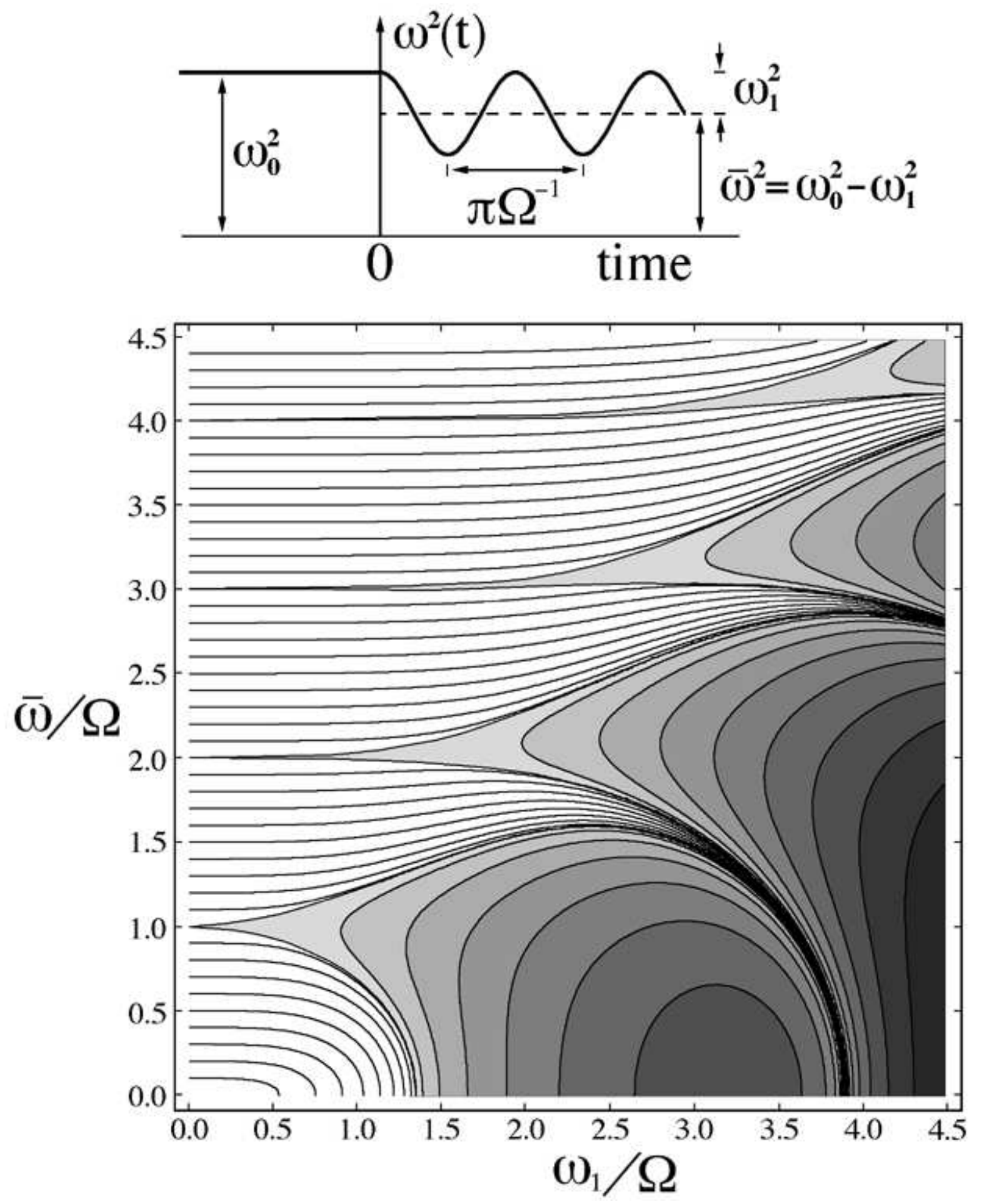


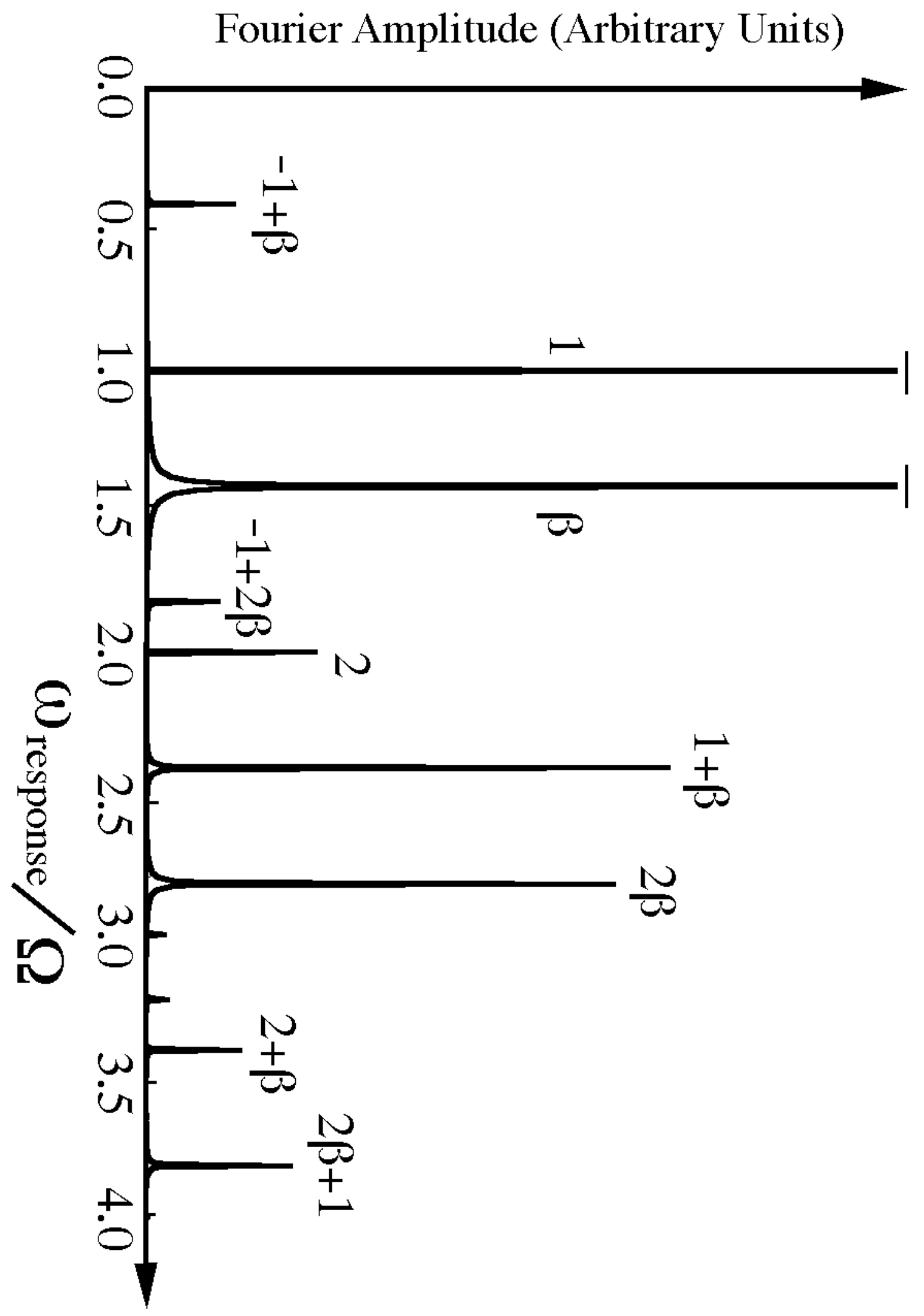

